\title{
Functions Like Convex Functions
}

\begin{abstract}
Zlatko Pavić
Mechanical Engineering Faculty in Slavonski Brod, University of Osijek, Trg Ivane Brlić Mažuranić 2, 35000 Slavonski Brod, Croatia
\end{abstract}

Correspondence should be addressed to Zlatko Pavić; zpavic@sfsb.hr

Received 30 July 2014; Accepted 6 October 2014

Academic Editor: Janusz Matkowski

Copyright (C) 2015 Zlatko Pavić. This is an open access article distributed under the Creative Commons Attribution License, which permits unrestricted use, distribution, and reproduction in any medium, provided the original work is properly cited.

The paper deals with convex sets, functions satisfying the global convexity property, and positive linear functionals. Jensen's type inequalities can be obtained by using convex combinations with the common center. Following the idea of the common center, the functional forms of Jensen's inequality are considered in this paper.

\section{Introduction}

Introduction is intended to be a brief overview of the concept of convexity and affinity. Let $\mathbb{X}$ be a real linear space. Let $a, b \in$ $\mathbb{X}$ be points and let $\alpha, \beta \in \mathbb{R}$ be coefficients. Their binomial combination

$$
\alpha a+\beta b
$$

is convex if $\alpha, \beta \geq 0$ and if

$$
\alpha+\beta=1 .
$$

If $c=\alpha a+\beta b$, then the point $c$ itself is called the combination center.

A set $\mathcal{S} \subseteq \mathbb{X}$ is convex if it contains all binomial convex combinations of its points. The convex hull conv $\mathcal{S}$ of the set $\mathcal{S}$ is the smallest convex set containing $\mathcal{S}$, and it consists of all binomial convex combinations of points of $\mathcal{S}$.

Let $\mathscr{C} \subseteq \mathbb{X}$ be a convex set. A function $f: \mathscr{C} \rightarrow \mathbb{R}$ is convex if the inequality

$$
f(\alpha a+\beta b) \leq \alpha f(a)+\beta f(b)
$$

holds for all binomial convex combinations $\alpha a+\beta b$ of pairs of points $a, b \in \mathscr{C}$.

Requiring only the condition in (2) for coefficients and requiring the equality in (3), we get a characterization of the affinity.

Implementing mathematical induction, we can prove that all of the above applies to $n$-membered combinations for any positive integer $n$. In that case, the inequality in (3) is the famous Jensen's inequality obtained in [1]. Numerous papers have been written on Jensen's inequality; different types and variants can be found in $[2,3]$.

\section{Positive Linear Functionals and Convex Sets of Functions}

Let $\mathscr{X}$ be a nonempty set, and let $\mathbb{X}$ be a subspace of the linear space of all real functions on the domain $\mathscr{X}$. We assume that $\mathbb{X}$ contains the unit function $\mathbf{1}$ defined by $\mathbf{1}(x)=1$ for every $x \in \mathscr{X}$.

Let $\mathscr{I} \subseteq \mathbb{R}$ be an interval, and let $\mathbb{X}_{\mathcal{F}}$ be the set containing all functions $g \in \mathbb{X}$ with the image in $\mathscr{I}$. Then, $\mathbb{X}_{\mathscr{I}}$ is convex set in the space $\mathbb{X}$. The same is true for convex sets of Euclidean spaces. Let $\mathscr{C} \subseteq \mathbb{R}^{k}$ be a convex set, and let $\left(\mathbb{X}^{k}\right)_{\mathscr{C}}$ be the set containing all function $k$-tuples $g=\left(g_{1}, \ldots, g_{k}\right) \in$ $\mathbb{X}^{k}$ with the image in $\mathscr{C}$. Then, $\left(\mathbb{X}^{k}\right)_{\mathscr{C}}$ is convex set in the space $\mathbb{X}^{k}$.

A linear functional $L: \mathbb{X} \rightarrow \mathbb{R}$ is positive (nonnegative) if $L(g) \geq 0$ for every nonnegative function $g \in \mathbb{X}$, and $L$ is unital (normalized) if $L(\mathbf{1})=1$. If $g \in \mathbb{X}$, then for every unital positive functional $L$ the number $L(g)$ is in the closed interval of real numbers containing the image of $g$. Through the paper, the space of all linear functionals on the space $\mathbb{X}$ will be denoted with $\mathbb{L}(\mathbb{X})$.

Let $f: \mathbb{R} \rightarrow \mathbb{R}$ be an affine function, that is, the function of the form $f(x):=\kappa x+\lambda$ where $\kappa$ and $\lambda$ are real constants. 
If $g_{1}, \ldots, g_{n} \in \mathbb{X}$ are functions and if $L_{1}, \ldots, L_{n} \in \mathbb{L}(\mathbb{X})$ are positive functionals providing the unit equality

$$
\sum_{i=1}^{n} L_{i}(\mathbf{1})=1 \text {, }
$$

then

$$
\begin{aligned}
f\left(\sum_{i=1}^{n} L_{i}\left(g_{i}\right)\right) & =\kappa \sum_{i=1}^{n} L_{i}\left(g_{i}\right)+\lambda \sum_{i=1}^{n} L_{i}(\mathbf{1})=\sum_{i=1}^{n} L_{i}\left(\kappa g_{i}+\lambda \mathbf{1}\right) \\
& =\sum_{i=1}^{n} L_{i}\left(f\left(g_{i}\right)\right) .
\end{aligned}
$$

Respecting the requirement of unit equality in (4), the sum $\sum_{i=1}^{n} L_{i}\left(g_{i}\right)$ could be called the functional convex combination. In the case $n=1$, the functional $L=L_{1}$ must be unital by the unit equality in (4).

In 1931, Jessen stated the functional form of Jensen's inequality for convex functions of one variable; see [4]. Adapted to our purposes, that statement is as follows.

Theorem A. Let $\mathscr{I} \subseteq \mathbb{R}$ be a closed interval, and let $g \in \mathbb{X}_{\mathscr{I}}$ be a function.

Then, a unital positive functional $L \in \mathbb{L}(\mathbb{X})$ ensures the inclusion

$$
L(g) \in \mathscr{I}
$$

and satisfies the inequality

$$
f(L(g)) \leq L(f(g))
$$

for every continuous convex function $f: \mathscr{I} \rightarrow \mathbb{R}$ providing that $f(g) \in \mathbb{X}$.

If $f$ is concave, then the reverse inequality is valid in (7). If $f$ is affine, then the equality is valid in (7).

The interval $\mathscr{I}$ must be closed, otherwise it could happen that $L(g) \notin \mathscr{I}$. The function $f$ must be continuous, otherwise it could happen that the inequality in (7) does not apply. Such boundary cases are presented in [5].

In 1937, McShane extended the functional form of Jensen's inequality to convex functions of several variables. He has covered the generalization in two steps, calling them the geometric (the inclusion in (8)) and analytic (the inequality in (9)) formulation of Jensen's inequality; see [6, Theorems 1 and 2]. Summarized in a theorem, that generalization is as follows.

Theorem B. Let $\mathscr{C} \subseteq \mathbb{R}^{k}$ be a closed convex set, and let $g=$ $\left(g_{1}, \ldots, g_{k}\right) \in\left(\mathbb{X}^{k}\right)_{\mathscr{C}}$ be a function.

Then, a unital positive functional $L \in \mathbb{L}(\mathbb{X})$ ensures the inclusion

$$
\left(L\left(g_{1}\right), \ldots, L\left(g_{k}\right)\right) \in \mathscr{C}
$$

and satisfies the inequality

$$
f\left(L\left(g_{1}\right), \ldots, L\left(g_{k}\right)\right) \leq L\left(f\left(g_{1}, \ldots, g_{k}\right)\right)
$$

for every continuous convex function $f: \mathscr{C} \rightarrow \mathbb{R}$ providing that $f\left(g_{1}, \ldots, g_{k}\right) \in \mathbb{X}$.

If $f$ is concave, then the reverse inequality is valid in (9). If $f$ is affine, then the equality is valid in (9).

\section{Main Results}

3.1. Functions of One Variable. The main result of this subsection is Theorem 1 relying on the idea of a convex function graph and its secant line. Using functions that are more general than convex functions and positive linear functionals, we obtain the functional Jensen's type inequalities.

Through the paper, we will use an interval $\mathscr{I} \subseteq \mathbb{R}$ and a bounded closed subinterval $[a, b] \subseteq \mathscr{I}$ with endpoints $a<b$.

Every number $x \in \mathbb{R}$ can be uniquely presented as the binomial affine combination

$$
x=\frac{b-x}{b-a} a+\frac{x-a}{b-a} b,
$$

which is convex if and only if the number $x$ belongs to the interval $[a, b]$. Let $f: \mathscr{I} \rightarrow \mathbb{R}$ be a function, and let $f_{\{a, b\}}^{\text {line }}: \mathbb{R} \rightarrow \mathbb{R}$ be the function of the line passing through the points $A(a, f(a))$ and $B(b, f(b))$ of the graph of $f$. Applying the affinity of the function $f_{\{a, b\}}^{\text {line }}$ to the combination in (10), we obtain its equation

$$
f_{\{a, b\}}^{\text {line }}(x)=\frac{b-x}{b-a} f(a)+\frac{x-a}{b-a} f(b) .
$$

The consequence of the representations in (10) and (11) is the fact that every convex function $f: \mathscr{I} \rightarrow \mathbb{R}$ satisfies the inequality

$$
f(x) \leq f_{\{a, b\}}^{\text {line }}(x) \quad \text { for } x \in[a, b]
$$

and the reverse inequality

$$
f(x) \geq f_{\{a, b\}}^{\text {line }}(x) \quad \text { for } x \in \mathscr{I} \backslash(a, b) .
$$

In the following consideration, we use continuous functions satisfying the inequalities in (12)-(13).

Theorem 1. Let $\mathscr{I} \subseteq \mathbb{R}$ be a closed interval, let $[a, b] \subseteq \mathscr{I}$ be $a$ bounded closed subinterval, and let $g \in \mathbb{X}_{[a, b]}$ and $h \in \mathbb{X}_{\mathscr{I} \backslash(a, b)}$ be functions. that

Then, a pair of unital positive functionals $L, H \in \mathbb{L}(\mathbb{X})$ such

$$
L(g)=H(h),
$$

satisfies the inequality

$$
L(f(g)) \leq H(f(h))
$$

for every continuous function $f: \mathscr{I} \rightarrow \mathbb{R}$ satisfying (12)-(13) and providing that $f(g), f(h) \in \mathbb{X}$.

Proof. The number $L(g)$ belongs to the interval $[a, b]$ by the inclusion in (6). Using the features of the function $f$ and applying the affinity of the function $f_{\{a, b\}}^{\text {line }}$, we get

$$
\begin{aligned}
L(f(g)) & \leq L\left(f_{\{a, b\}}^{\text {line }}(g)\right)=f_{\{a, b\}}^{\text {line }}(L(g)) \\
& =f_{\{a, b\}}^{\text {line }}(H(h))=H\left(f_{\{a, b\}}^{\text {line }}(h)\right) \\
& \leq H(f(h))
\end{aligned}
$$

because $f_{\{a, b\}}^{\text {line }}(h(x)) \leq f(h(x))$ for every $x \in \mathscr{X}$. 
It is obvious that a continuous convex function $f: \mathscr{I} \rightarrow$ $\mathbb{R}$ satisfies Theorem 1 for every subinterval $[a, b] \subseteq \mathscr{I}$ with endpoints $a<b$. The function used in Theorem 1 is shown in Figure 1. Such a function satisfies only the global property of convexity on the sets $[a, b]$ and $\mathscr{I} \backslash(a, b)$.

Involving the binomial convex combination $\alpha a+\beta b$ with the equality in (14) by assuming that

$$
L(g)=\alpha a+\beta b=H(h)
$$

and inserting the term $\alpha f(a)+\beta f(b)$ in (16) via the double equality

$$
f_{\{a, b\}}^{\text {line }}(L(g))=\alpha f(a)+\beta f(b)=f_{\{a, b\}}^{\text {line }}(H(h))
$$

which is true because $f_{\{a, b\}}^{\text {line }}(\alpha a+\beta b)=\alpha f(a)+\beta f(b)$, we achieve the double inequality

$$
L(f(g)) \leq \alpha f(a)+\beta f(b) \leq H(f(h)) .
$$

The functions used in Theorem 1 satisfy the functional form of Jensen's inequality in the following case.

Corollary 2. Let $\mathscr{I} \subseteq \mathbb{R}$ be a closed interval, let $[a, b] \subseteq \mathscr{I}$ be a bounded closed subinterval, and let $h \in \mathbb{X}_{\mathscr{f} \backslash(a, b)}$ be a function.

Then, a unital positive functional $H \in \mathbb{L}(\mathbb{X})$ such that

$$
H(h) \in[a, b]
$$

satisfies the inequality

$$
f(H(h)) \leq H(f(h))
$$

for every continuous function satisfying (12)-(13) and providing that $f(h) \in \mathbb{X}$.

Proof. Putting $\alpha a+\beta b=H(h)$, it follows that

$$
\begin{aligned}
f(H(h)) & =f(\alpha a+\beta b) \leq f_{\{a, b\}}^{\text {line }}(\alpha a+\beta b) \\
& =\alpha f(a)+\beta f(b) \leq H(f(h))
\end{aligned}
$$

by the right inequality in (19).

Now, we give a characterization of continuous convex functions by using unital positive functionals.

Proposition 3. Let $\mathscr{I} \subseteq \mathbb{R}$ be a closed interval. A continuous function $f: \mathscr{I} \rightarrow \mathbb{R}$ is convex if and only if it satisfies the inequality

$$
L(f(g)) \leq f_{\{a, b\}}^{\text {line }}(L(g))
$$

for every pair of interval endpoints $a, b \in \mathscr{I}$, every function $g \in$ $\mathbb{X}_{[a, b]}$ such that $f(g) \in \mathbb{X}$, and every unital positive functional $L \in \mathbb{L}(\mathbb{X})$.

Proof. Let us prove the sufficiency. Let $c:=\alpha a+\beta b$ be a convex combination of points $a, b \in \mathscr{I}$ where $a<b$. We take the constant function $g=c \mathbf{1}$ in $\mathbb{X}_{[a, b]}$ (actually $g(x)=c$

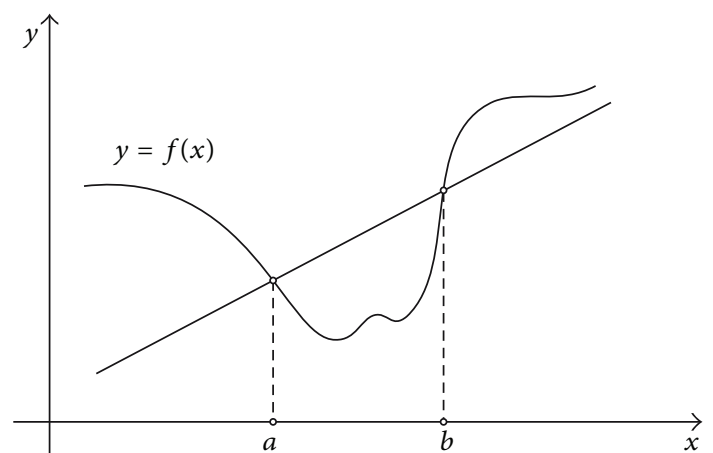

FIGURE 1: A continuous function satisfying (12)-(13).

for every $x \in \mathscr{X}$ ) and a unital positive functional $L$. Then, connecting

$$
\begin{gathered}
L(f(g))=L(f(c) \mathbf{1})=f(c)=f(\alpha a+\beta b), \\
f_{\{a, b\}}^{\text {line }}(L(g))=f_{\{a, b\}}^{\text {line }}(\alpha a+\beta b)=\alpha f(a)+\beta f(b)
\end{gathered}
$$

via (23), we get the convexity inequality in (3).

3.2. Functions of Several Variables. We want to transfer the results of the previous subsection to higher dimensions. The main result in this subsection is Theorem 6 generalizing Theorem 1 to functions of several variables.

Let $\mathscr{C} \subseteq \mathbb{R}^{2}$ be a convex set, let $\triangle \subseteq \mathscr{C}$ be a triangle with vertices $A, B$, and $C$, and let $\triangle^{o}$ be its interior. In the following observation, we assume that $f: \mathscr{C} \rightarrow \mathbb{R}$ is a continuous function satisfying the inequality

$$
f(P) \leq f_{\{A, B, C\}}^{\text {plane }}(P) \quad \text { for } P \in \triangle
$$

and the reverse inequality

$$
f(P) \geq f_{\{A, B, C\}}^{\text {plane }}(P) \quad \text { for } P \in \mathscr{C} \backslash \triangle^{o},
$$

where $f_{\{A, B, C\}}^{\text {plane }}$ is the function of the plane passing through the corresponding points of the graph of $f$.

It should be noted that convex functions of two variables do not generally satisfy (26). The next example confirms this claim.

Example 4. We take the convex function $f(x, y)=x^{2}+y^{2}$, the triangle with vertices $A(0,0), B(1,0)$, and $C(0,2)$, and the outside point $P(1,1)$.

The valuation of functions $f$ and $f_{\{A, B, C\}}^{\text {plane }}(x, y)=x+2 y$ at the point $P$ is

$$
2=f(P)<f_{\{A, B, C\}}^{\text {plane }}(P)=3
$$

as opposed to (26).

The generalization of Theorem 1 to two dimensions is as follows. 
Lemma 5. Let $\mathscr{C} \subseteq \mathbb{R}^{2}$ be a closed convex set, let $\triangle \subseteq \mathscr{C}$ be a triangle, and let $g=\left(g_{1}, g_{2}\right) \in\left(\mathbb{X}^{2}\right)_{\triangle}$ and $h=\left(h_{1}, h_{2}\right) \in$ $\left(\mathbb{X}^{2}\right)_{\mathscr{C} \backslash \triangle^{\circ}}$ be functions. that

Then, a pair of unital positive functionals $L, H \in \mathbb{L}(\mathbb{X})$ such

$$
\left(L\left(g_{1}\right), L\left(g_{2}\right)\right)=\left(H\left(h_{1}\right), H\left(h_{2}\right)\right)
$$

satisfies the inequality

$$
L\left(f\left(g_{1}, g_{2}\right)\right) \leq H\left(f\left(h_{1}, h_{2}\right)\right)
$$

for every continuous function satisfying (25)-(26) and providing that $f\left(g_{1}, g_{2}\right), f\left(h_{1}, h_{2}\right) \in \mathbb{X}$.

Proof. The proof is similar to that of Theorem 1. Using the triangle vertices $A, B$, and $C$, we apply the plane function $f_{\{A, B, C\}}^{\text {plane }}$ instead of the line function $f_{\{a, b\}}^{\text {line }}$.

The previous lemma suggests how the results of the previous subsection can be transferred to higher dimensions.

Let $S_{1}, \ldots, S_{k+1} \in \mathbb{R}^{k}$ be points. Their convex hull

$$
\mathcal{S}=\operatorname{conv}\left\{S_{1}, \ldots, S_{k+1}\right\}
$$

is the $k$-simplex in $\mathbb{R}^{k}$ if the points $S_{1}-S_{k+1}, \ldots, S_{k}-S_{k+1}$ are linearly independent.

Let $\mathscr{C} \subseteq \mathbb{R}^{k}$ be a convex set, and let $\mathcal{S} \subseteq \mathscr{C}$ be a $k$-simplex with vertices $S_{1}, \ldots, S_{k+1}$. In the consideration that follows, we use a function $f: \mathscr{C} \rightarrow \mathbb{R}$ satisfying the inequality

$$
f(P) \leq f_{\left\{S_{1}, \ldots, S_{k+1}\right\}}^{\text {hyperplane }}(P) \quad \text { for } P \in \mathcal{S}
$$

and the reverse inequality

$$
f(P) \geq f_{\left\{S_{1}, \ldots, S_{k+1}\right\}}^{\text {hyperplane }}(P) \quad \text { for } P \in \mathscr{C} \backslash \mathcal{S}^{o},
$$

where $f_{\left\{S_{1}, \ldots, S_{k+1}\right\}}^{\text {hyperlane }}$ is the function of the hyperplane passing through the corresponding points of the graph of $f$.

Theorem 6. Let $\mathscr{C} \subseteq \mathbb{R}^{k}$ be a closed convex set, let $\mathcal{S} \subseteq \mathscr{C}$ be a $k$-simplex, and let $g=\left(g_{1}, \ldots, g_{k}\right) \in\left(\mathbb{X}^{k}\right)_{\mathcal{S}}$ and $h=$ $\left(h_{1}, \ldots, h_{k}\right) \in\left(\mathbb{X}^{k}\right)_{\mathscr{C} \backslash \delta^{o}}$ be functions. that

Then, a pair of unital positive functionals $L, H \in \mathbb{L}(\mathbb{X})$ such

$$
\left(L\left(g_{1}\right), \ldots, L\left(g_{k}\right)\right)=\left(H\left(h_{1}\right), \ldots, H\left(h_{k}\right)\right)
$$

satisfies the inequality

$$
L\left(f\left(g_{1}, \ldots, g_{k}\right)\right) \leq H\left(f\left(h_{1}, \ldots, h_{k}\right)\right)
$$

for every continuous function satisfying (31)-(32) and providing that $f\left(g_{1}, \ldots, g_{k}\right), f\left(h_{1}, \ldots, h_{k}\right) \in \mathbb{X}$.

Proof. Relying on the hyperplane function $f_{\left\{S_{1}, \ldots, S_{k+1}\right\}}^{\text {hyperplane }}$ where $S_{1}, \ldots, S_{k+1}$ are the simplex vertices, we can apply the proof similar to that of Theorem 1.
Including the $(k+1)$-membered convex combination $\sum_{p=1}^{k+1} \gamma_{p} S_{p}$ with the equality in (33) in a way that

$$
\left(L\left(g_{1}\right), \ldots, L\left(g_{k}\right)\right)=\sum_{p=1}^{k+1} \gamma_{p} S_{p}=\left(H\left(h_{1}\right), \ldots, H\left(h_{k}\right)\right)
$$

and using the double equality

$$
\begin{aligned}
& f_{\left\{S_{1}, \ldots, S_{k+1}\right\}}^{\text {hyperlane }}\left(L\left(g_{1}\right), \ldots, L\left(g_{k}\right)\right) \\
& \quad=\sum_{p=1}^{k+1} \gamma_{p} f\left(s_{p}\right) \\
& \quad=f_{\left\{S_{1}, \ldots, S_{k+1}\right\}}^{\text {hyperplane }}\left(H\left(h_{1}\right), \ldots, H\left(h_{k}\right)\right),
\end{aligned}
$$

we can derive the double inequality

$$
L\left(f\left(g_{1}, \ldots, g_{k}\right)\right) \leq \sum_{p=1}^{k+1} \gamma_{p} f\left(S_{p}\right) \leq H\left(f\left(h_{1}, \ldots, h_{k}\right)\right) .
$$

The following functional form of Jensen's inequality is true for functions of several variables.

Corollary 7. Let $\mathscr{C} \subseteq \mathbb{R}^{k}$ be a closed convex set, let $\mathcal{S} \subseteq \mathscr{C}$ be a $k$-simplex, and let $h=\left(h_{1}, \ldots, h_{k}\right) \in\left(\mathbb{X}^{k}\right)_{\mathscr{C} \backslash \mathcal{S}^{\circ}}$ be a function.

Then, a unital positive functional $H \in \mathbb{L}(\mathbb{X})$ such that

$$
\left(H\left(h_{1}\right), \ldots, H\left(h_{k}\right)\right) \in \mathcal{S}
$$

satisfies the inequality

$$
f\left(H\left(h_{1}\right), \ldots, H\left(h_{k}\right)\right) \leq H\left(f\left(h_{1}, \ldots, h_{k}\right)\right)
$$

for every continuous function satisfying (25)-(26) and providing that $f\left(h_{1}, \ldots, h_{k}\right) \in \mathbb{X}$.

Continuous convex functions of several variables can be characterized by unital positive functionals in the following way. The dimension of a convex set is defined as the dimension of its affine hull.

Proposition 8. Let $\mathscr{C} \subseteq \mathbb{R}^{k}$ be a closed convex set of dimension $k$. A continuous function $f: \mathscr{C} \rightarrow \mathbb{R}$ is convex if and only if it satisfies the inequality

$$
L\left(f\left(g_{1}, \ldots, g_{k}\right)\right) \leq f_{\left\{S_{1}, \ldots, S_{k+1}\right\}}^{\text {hyperplane }}\left(L\left(g_{1}\right), \ldots, L\left(g_{k}\right)\right)
$$

for every $(k+1)$-tuple of $k$-simplex vertices $S_{1}, \ldots, S_{k+1} \in$ $\mathscr{C}$, every function $g=\left(g_{1}, \ldots, g_{k}\right) \in\left(\mathbb{X}^{k}\right)_{\mathcal{S}}$ such that $f\left(g_{1}, \ldots, g_{k}\right) \in \mathbb{X}$, and every unital positive functional $L \in$ $\mathbb{L}(\mathbb{X})$.

Proof. To prove the sufficiency, we take a convex combination $C=\sum_{p=1}^{k+1} \gamma_{p} S_{p}$ of $k$-simplex vertices $S_{1}, \ldots, S_{k+1} \in \mathscr{C}$. If $C=$ $\left(c_{1}, \ldots, c_{k}\right)$, we take the constant mapping $g=\left(g_{1}, \ldots, g_{k}\right) \in$ $\left(\mathbb{X}^{k}\right)_{\mathcal{\delta}}$ consisting of constant functions $g_{i}=c_{i} \mathbf{1}$ and continue 
the proof in the same way as in Proposition 3. Finally, we get Jensen's inequality

$$
f\left(\sum_{p=1}^{k+1} \gamma_{p} S_{p}\right) \leq \sum_{p=1}^{k+1} \gamma_{p} f\left(S_{p}\right)
$$

confirming the convexity of the function $f$.

\section{Applications to Functional Quasiarithmetic Means}

Functions investigated in Subsection 3.1 can be included to quasiarithmetic means by applying methods such as those for convex functions. The basic facts relating to quasiarithmetic and power means can be found in [7]. For more details on different forms of quasiarithmetic and power means, as well as their refinements, see [8].

The next generalization of Theorem 1 will be applied to the consideration of functional quasiarithmetic means.

Corollary 9. Let $\mathscr{I} \subseteq \mathbb{R}$ be a closed interval, let $[a, b] \subseteq \mathscr{I}$ be a bounded closed subinterval, and let $g_{1}, \ldots, g_{n} \in \mathbb{X}_{[a, b]}$ and $h_{1}, \ldots, h_{m} \in \mathbb{X}_{\mathscr{F} \backslash(a, b)}$ be functions.

Then, a pair of collections of positive functionals $L_{i}, H_{j} \in$ $\mathbb{L}(\mathbb{X})$ providing the unit equalities $\sum_{i=1}^{n} L_{i}(\mathbf{1})=\sum_{j=1}^{m} H_{j}(\mathbf{1})=$ 1 and the equality

$$
\sum_{i=1}^{n} L_{i}\left(g_{i}\right)=\sum_{j=1}^{m} H_{j}\left(h_{j}\right)
$$

satisfies the inequality

$$
\sum_{i=1}^{n} L_{i}\left(f\left(g_{i}\right)\right) \leq \sum_{j=1}^{m} H_{j}\left(f\left(h_{j}\right)\right)
$$

for every continuous function satisfying (12)-(13) and providing that all functions $f\left(g_{i}\right), f\left(h_{j}\right) \in \mathbb{X}$.

Now, we present a way of introducing the functional quasiarithmetic means. Let $g_{1}, \ldots, g_{n} \in \mathbb{X}_{\mathscr{I}}$ be functions, and let $\varphi: \mathscr{I} \rightarrow \mathbb{R}$ be a strictly monotone continuous function such that all $\varphi\left(g_{i}\right) \in \mathbb{X}$. Let $L_{1}, \ldots, L_{n}: \mathbb{X} \rightarrow$ $\mathbb{R}$ be positive linear functionals providing the unit equality $\sum_{i=1}^{n} L_{i}(\mathbf{1})=1$. The quasiarithmetic mean of functions $g_{i}$ respecting the function $\varphi$ and functionals $L_{i}$ can be defined by

$$
M_{\varphi}\left(L_{1} \ldots, L_{n} ; g_{1}, \ldots, g_{n}\right)=\varphi^{-1}\left(\sum_{i=1}^{n} L_{i}\left(\varphi\left(g_{i}\right)\right)\right) .
$$

In what follows, we will use the abbreviation $M_{\varphi}\left(L_{i}, g_{i}\right)$ for the above mean. The term in parentheses belongs to the interval $\varphi(\mathscr{I})$, and therefore the quasiarithmetic mean $M_{\varphi}\left(L_{i}, g_{i}\right)$ belongs to the interval $\mathscr{I}$.

In applications of the function convexity, we use a pair of strictly monotone continuous functions $\varphi, \psi: \mathscr{I} \rightarrow \mathbb{R}$ such that $\psi$ is convex with respect to $\varphi$ (it also says that $\psi$ is $\varphi$-convex), which means that the function $f=\psi\left(\varphi^{-1}\right)$ is convex on the interval $\varphi(\mathscr{I})$. A similar notation is used for the concavity.

Instead of the convexity of $f$, we will apply the conditions in (12)-(13) via Corollary 9 as follows.

Theorem 10. Let $\mathscr{I} \subseteq \mathbb{R}$ be a closed interval, let $[a, b] \subseteq \mathscr{I}$ be a bounded closed subinterval, and let $g_{1}, \ldots, g_{n} \in \mathbb{X}_{[a, b]}$ and $h_{1}, \ldots, h_{m} \in \mathbb{X}_{\mathscr{I} \backslash(a, b)}$ be functions. Let $L_{i}, H_{j} \in \mathbb{L}(\mathbb{X})$ be a pair of collections of positive functionals providing the unit equalities $\sum_{i=1}^{n} L_{i}(1)=\sum_{j=1}^{m} H_{j}(1)=1$. Let $\varphi, \psi: \mathscr{I} \rightarrow \mathbb{R}$ be strictly monotone continuous functions such that all functions $\varphi\left(g_{i}\right), \varphi\left(h_{j}\right), \psi\left(g_{i}\right), \psi\left(h_{j}\right) \in \mathbb{X}$, and let $f=\psi\left(\varphi^{-1}\right)$ be the composite function.

If $f$ satisfies (12)-(13) and $\psi$ is increasing and if the equality

$$
M_{\varphi}\left(L_{i}, g_{i}\right)=M_{\varphi}\left(H_{j}, h_{j}\right)
$$

is valid, then we have the inequality

$$
M_{\psi}\left(L_{i}, g_{i}\right) \leq M_{\psi}\left(H_{j}, h_{j}\right) .
$$

Proof. We take $\mathscr{J}=\varphi(\mathscr{I})$ and $[c, d]=\varphi([a, b])$. We will apply Corollary 9 to the functions $u_{i}=\varphi\left(g_{i}\right) \in \mathbb{X}_{[c, d]}$ and $v_{j}=$ $\varphi\left(h_{j}\right) \in \mathbb{X}_{\mathscr{F} \backslash(c, d)}$ and the function $f: \mathscr{J} \rightarrow \mathbb{R}$.

Using the equality $\varphi\left(M_{\varphi}\left(L_{i}, g_{i}\right)\right)=\varphi\left(M_{\varphi}\left(H_{j}, h_{j}\right)\right)$ and including the functions $u_{i}$ and $v_{j}$, we have

$$
\sum_{i=1}^{n} L_{i}\left(u_{i}\right)=\sum_{j=1}^{m} H_{j}\left(v_{j}\right) .
$$

Then, the inequality

$$
\sum_{i=1}^{n} L_{i}\left(f\left(u_{i}\right)\right) \leq \sum_{j=1}^{m} H_{j}\left(f\left(v_{j}\right)\right)
$$

follows from Corollary 9, and applying the increasing function $\psi^{-1}$, we get

$$
\psi^{-1}\left(\sum_{i=1}^{n} L_{i}\left(f\left(u_{i}\right)\right)\right) \leq \psi^{-1}\left(\sum_{j=1}^{m} H_{j}\left(f\left(v_{j}\right)\right)\right) .
$$

The above inequality is actually the inequality in (46) because $f\left(u_{i}\right)=\psi\left(g_{i}\right)$ and $f\left(v_{j}\right)=\psi\left(h_{j}\right)$.

All the cases of the above theorem are as follows.

Corollary 11. Let $f=\psi\left(\varphi^{-1}\right)$ be the composite function satisfying the conditions of Theorem 10.

If either $f$ satisfies (12)-(13) and $\psi$ is increasing or $-f$ satisfies (12)-(13) and $\psi$ is decreasing and if the equality in (45) is valid, then the inequality holds in (46).

If either $f$ satisfies (12)-(13) and $\psi$ is decreasing or $-f$ satisfies (12)-(13) and $\psi$ is increasing and if the equality in (45) is valid, then the reverse inequality holds in (46).

A special case of the quasiarithmetic means in (44) is power means depending on real exponents $r$. Thus, using the functions

$$
\varphi_{r}(x)= \begin{cases}x^{r}, & r \neq 0 \\ \ln x, & r=0\end{cases}
$$


where $x \in(0, \infty)$, we get the power means of order $r$ in the form

$$
M_{r}\left(L_{i}, g_{i}\right)= \begin{cases}\left(\sum_{i=1}^{n} L_{i}\left(g_{i}^{r}\right)\right)^{1 / r}, & r \neq 0 \\ \exp \left(\sum_{i=1}^{n} L_{i}\left(\ln g_{i}\right)\right), & r=0 .\end{cases}
$$

To apply Theorem 1 to the power means, we use a closed interval $\mathscr{I}=[\varepsilon, \infty)$ where $\varepsilon$ is a positive number and the equality

$$
M_{1}\left(L, g_{i}\right)=\sum_{i=1}^{n} L_{i}\left(g_{i}\right) .
$$

Corollary 12. Let $\mathscr{I}=[\varepsilon, \infty)$ be an unbounded closed interval where $\varepsilon>0$, let $[a, b] \subset \mathscr{I}$ be a bounded closed subinterval, and let $g_{1}, \ldots, g_{n} \in \mathbb{X}_{[a, b]}$ and $h_{1}, \ldots, h_{m} \in$ $\mathbb{X}_{\mathscr{I} \backslash(a, b)}$ be functions. Let $L_{i}, H_{j} \in \mathbb{L}(\mathbb{X})$ be a pair of collections of positive functionals providing the unit equalities $\sum_{i=1}^{n} L_{i}(1)=$ $\sum_{j=1}^{m} H_{j}(1)=1$.

If

$$
M_{1}\left(L_{i}, g_{i}\right)=M_{1}\left(H_{j}, h_{j}\right)
$$

then

$$
\begin{array}{ll}
M_{r}\left(L_{i}, g_{i}\right) \leq M_{r}\left(H_{j}, h_{j}\right) & \text { for } r \geq 1, \\
M_{r}\left(L_{i}, g_{i}\right) \geq M_{r}\left(H_{j}, h_{j}\right) & \text { for } r \leq 1
\end{array}
$$

Proof. The proof follows from Theorem 10 and Corollary 11 by using convex and concave functions such as $\varphi(x)=x$ and $\psi(x)=x^{r}$ for $r \neq 0$, and $\psi(x)=\ln x$ for $r=0$.

\section{Applications to Discrete and Integral Inequalities}

Our aim is to use Theorem 6 to obtain certain discrete and integral inequalities concerning functions of several variables. The following is the application to discrete inequalities.

Proposition 13. Let $\mathscr{C} \subseteq \mathbb{R}^{k}$ be a closed convex set, let $\mathcal{S} \subseteq \mathscr{C}$ be a $k$-simplex, let $\sum_{i=1}^{n} \alpha_{i} A_{i}$ be a convex combination of points $A_{i} \in \mathcal{S}$, and let $\sum_{j=1}^{m} \beta_{j} B_{j}$ be a convex combination of points $B_{j} \in \mathscr{C} \backslash \mathcal{S}^{o}$.

If the above convex combinations have the common center

$$
\sum_{i=1}^{n} \alpha_{i} A_{i}=\sum_{j=1}^{m} \beta_{j} B_{j}
$$

then the inequality

$$
\sum_{i=1}^{n} \alpha_{i} f\left(A_{i}\right) \leq \sum_{j=1}^{m} \beta_{j} f\left(B_{j}\right)
$$

holds for every continuous function $f: \mathscr{C} \rightarrow \mathbb{R}$ satisfying (31)-(32).
Proof. We take the set $\mathscr{X}=\mathscr{C}$ and the space $\mathbb{X}$ containing all real functions on $\mathscr{C}$. We also take any simplex vertex $S$ and its coordinates $\left(s_{1}, \ldots, s_{k}\right)$.

Let $g_{p}, h_{p} \in \mathbb{X}(p=1, \ldots, k)$ be functions defined by

$$
\begin{aligned}
& g_{p}\left(x_{1}, \ldots, x_{k}\right)= \begin{cases}x_{p}, & \left(x_{1}, \ldots, x_{k}\right) \in \mathcal{S} \\
s_{p}, & \left(x_{1}, \ldots, x_{k}\right) \in \mathscr{C} \backslash \mathcal{S},\end{cases} \\
& h_{p}\left(x_{1}, \ldots, x_{k}\right)= \begin{cases}s_{p}, & \left(x_{1}, \ldots, x_{k}\right) \in \mathcal{S}^{o} \\
x_{p}, & \left(x_{1}, \ldots, x_{k}\right) \in \mathscr{C} \backslash \mathcal{S}^{o} .\end{cases}
\end{aligned}
$$

Then, $g=\left(g_{1}, \ldots, g_{k}\right) \in\left(\mathbb{X}^{k}\right)_{\mathcal{S}}$ and $h=\left(h_{1}, \ldots, h_{k}\right) \in$ $\left(\mathbb{X}^{k}\right)_{\mathscr{C} \backslash \mathcal{S}^{o}}$.

Let $L, H \in \mathbb{L}(\mathbb{X})$ be summarizing unital positive functionals defined by

$$
\begin{aligned}
& L(g)=\sum_{i=1}^{n} \alpha_{i} g\left(A_{i}\right), \\
& H(h)=\sum_{j=1}^{m} \beta_{j} h\left(B_{j}\right) .
\end{aligned}
$$

Applying the functional $L$ to the functions $g_{p}$ and the functional $H$ to the functions $h_{p}$, we obtain

$$
\begin{aligned}
\sum_{i=1}^{n} \alpha_{i} A_{i} & =\left(L\left(g_{1}\right), \ldots, L\left(g_{k}\right)\right) \\
& =\left(H\left(h_{1}\right), \ldots, H\left(h_{k}\right)\right)=\sum_{j=1}^{m} \beta_{j} B_{j} .
\end{aligned}
$$

Now, we can apply Theorem 6 and get the inequality

$$
\begin{aligned}
\sum_{i=1}^{n} \alpha_{i} f\left(A_{i}\right) & =L\left(f\left(g_{1}, \ldots, g_{k}\right)\right) \leq H\left(f\left(h_{1}, \ldots, h_{k}\right)\right) \\
& =\sum_{j=1}^{m} \beta_{j} f\left(B_{j}\right)
\end{aligned}
$$

which concludes the proof.

Proposition 13 does not generally hold for convex functions. The next example demonstrates a concrete planar case of $k=2$.

Example 14. We take the convex function $f(x, y)=x^{2}+y^{2}$, the triangle with vertices $A_{1}(-3,0), A_{2}(3,0)$, and $A_{3}(0,3)$, and the outside points $B_{1}(-2,2), B_{2}(0,-2)$, and $B_{3}(2,2)$.

Then, we have

$$
\begin{gathered}
\frac{1}{3} A_{1}+\frac{1}{3} A_{2}+\frac{1}{3} A_{3}=\frac{3}{8} B_{1}+\frac{2}{8} B_{2}+\frac{3}{8} B_{3}, \\
9=\frac{1}{3} f\left(A_{1}\right)+\frac{1}{3} f\left(A_{2}\right)+\frac{1}{3} f\left(A_{3}\right) \\
>\frac{3}{8} f\left(B_{1}\right)+\frac{2}{8} f\left(B_{2}\right)+\frac{3}{8} f\left(B_{3}\right)=7 .
\end{gathered}
$$


More details on the behavior of a convex function of two variables on the triangle and outside the triangle can be found in [9, Theorem 3.2]. Triangle cones have a prominent part in these considerations.

The integral analogy of the concept of convex combination is the concept of barycenter. Let $\mu$ be a positive measure on $\mathbb{R}^{k}$, and let $\mathscr{A} \subseteq \mathbb{R}^{k}$ be a $\mu$-measurable set with $\mu(\mathscr{A})>0$. Given the positive integer $n$, let $\mathscr{A}=\cup_{i=1}^{n} \mathscr{A}_{n i}$ be the partition of pairwise disjoint $\mu$-measurable sets $\mathscr{A}_{n i}$. Taking points $A_{n i} \in \mathscr{A}_{n i}$, we determine the convex combination

$$
A_{n}=\sum_{i=1}^{n} \frac{\mu\left(\mathscr{A}_{n i}\right)}{\mu(\mathscr{A})} A_{n i}
$$

whose center $A_{n}$ belongs to conv $\mathscr{A}$. The $\mu$-barycenter of the set $\mathscr{A}$ can be defined as the limit of the sequence $\left(A_{n}\right)_{n}$; that is,

$$
\begin{aligned}
M(\mathscr{A}, \mu) & =\lim _{n \rightarrow \infty}\left(\sum_{i=1}^{n} \frac{\mu\left(\mathscr{A}_{n i}\right)}{\mu(\mathscr{A})} A_{n i}\right) \\
& =\frac{1}{\mu(\mathscr{A})}\left(\int_{\mathscr{A}} x_{1} d \mu, \ldots, \int_{\mathscr{A}} x_{k} d \mu\right) .
\end{aligned}
$$

As defined above, the point $M(\mathscr{A}, \mu)$ is in $\operatorname{conv} \mathscr{A}$. So, the convex sets contain its barycenters. follows.

The application of Theorem 6 to integral inequalities is as

Proposition 15. Let $\mu$ be a positive measure on $\mathbb{R}^{k}$. Let $\mathscr{C} \subseteq$ $\mathbb{R}^{k}$ be a closed convex set, let $\mathcal{S} \subseteq \mathscr{C}$ be a $k$-simplex, and let $\mathscr{A} \subseteq \mathcal{S}$ and $\mathscr{B} \subseteq \mathscr{C} \backslash \mathcal{S}^{\circ}$ be sets of positive $\mu$-measures.

If the above sets have the common $\mu$-barycenter

$$
M(\mathscr{A}, \mu)=M(\mathscr{B}, \mu),
$$

then the inequality

$$
\frac{1}{\mu(\mathscr{A})} \int_{\mathscr{A}} f\left(x_{1}, \ldots, x_{k}\right) d \mu \leq \frac{1}{\mu(\mathscr{B})} \int_{\mathscr{B}} f\left(x_{1}, \ldots, x_{k}\right) d \mu
$$

holds for every continuous function $f: \mathscr{C} \rightarrow \mathbb{R}$ satisfying (31)-(32).

Proof. The proof is similar to that of Proposition 13 by using $\mathbb{X}$ as the space of all $\mu$-integrable functions on $\mathscr{C}$. We apply the integrating unital positive functional $L$ defined by

$$
L(g)=\frac{1}{\mu(\mathscr{A})} \int_{\mathscr{A}} g\left(x_{1}, \ldots, x_{k}\right) d \mu
$$

to the functions $g_{p}$ of (57), as well as the integrating unital positive functional $H$ defined by

$$
H(h)=\frac{1}{\mu(\mathscr{B})} \int_{\mathscr{B}} h\left(x_{1}, \ldots, x_{k}\right) d \mu
$$

to the functions $h_{p}$ of (58).
If $S_{1}, \ldots, S_{k+1}$ are the simplex vertices, then using the unique convex combination $\sum_{p=1}^{k+1} \gamma_{p} S_{p}$ satisfying

$$
M(\mathscr{A}, \mu)=\sum_{p=1}^{k+1} \gamma_{p} S_{p}=M(\mathscr{B}, \mu)
$$

and applying (37), we obtain the extension of (66) as the double inequality

$$
\begin{aligned}
\frac{1}{\mu(\mathscr{A})} \int_{\mathscr{A}} f\left(x_{1}, \ldots, x_{k}\right) d \mu & \leq \sum_{p=1}^{k+1} \gamma_{p} f\left(s_{p}\right) \\
& \leq \frac{1}{\mu(\mathscr{B})} \int_{\mathscr{B}} f\left(x_{1}, \ldots, x_{k}\right) d \mu .
\end{aligned}
$$

The above inequality is reminiscent of Hermite-Hadamard's inequality where discrete and integral terms are replaced, see the below inequality in (72).

Implementing convex combinations to the integral method, one may derive the following version of the HermiteHadamard inequality for convex functions on simplexes.

Proposition 16. Let $\mu$ be a positive measure on $\mathbb{R}^{k}$. Let $\mathcal{S} \subset \mathbb{R}^{k}$ be a $k$-simplex of positive $\mu$-measure, let $S_{1}, \ldots, S_{k+1}$ be simplex vertices, and let $\sum_{p=1}^{k+1} \gamma_{p} S_{p}$ be their convex combination.

If the convex combination center and the $\mu$-barycenter of $\mathcal{S}$ both fall at the same point

$$
\sum_{p=1}^{k+1} \gamma_{p} S_{p}=M(\mathcal{S}, \mu)
$$

then the double inequality

$$
f\left(\sum_{p=1}^{k+1} \gamma_{p} S_{p}\right) \leq \frac{1}{\mu(\mathcal{S})} \int_{\mathcal{S}} f\left(x_{1}, \ldots, x_{k}\right) d \mu \leq \sum_{p=1}^{k+1} \gamma_{p} f\left(S_{p}\right)
$$

holds for every $\mu$-integrable convex function $f: \delta \rightarrow \mathbb{R}$.

More on the important and interesting Hermite-Hadamard's inequality, including historical facts about its name, can be found in $[10,11]$.

\section{Conflict of Interests}

The author declares that there is no conflict of interests regarding the publication of this paper.

\section{Acknowledgments}

This work has been fully supported by Mechanical Engineering Faculty in Slavonski Brod and Croatian Science Foundation under Project 5435. The author thanks Velimir Pavić (graphic designer at Školska knjiga Zagreb) who has graphically prepared Figure 1. 


\section{References}

[1] J. L. W. V. Jensen, “Om konvekse Funktioner og Uligheder mellem Middelværdier," Nyt Tidsskrift for Matematik B, vol. 16, pp. 49-68, 1905.

[2] M. S. Moslehian and M. Kian, "Jensen type inequalities for Qclass functions," Bulletin of the Australian Mathematical Society, vol. 85, no. 1, pp. 128-142, 2012.

[3] Z. Pavić, J. Pečarić, and I. Perić, "Integral, discrete and functional variants of Jensen's inequality," Journal of Mathematical Inequalities, vol. 5, no. 2, pp. 253-264, 2011.

[4] B. Jessen, "Bemærkninger om konvekse Funktioner og Uligheder imellem Middelværdier. I," Matematisk Tidsskrift B, vol. 2, pp. 17-28, 1931.

[5] I. Rasa, "A note on Jensen's inequality," in Itinerant Seminar on Functional Equations, Approximation and Convexity, pp. 275280, Universitatea Babes-Bolyai, Cluj-Napoca, Romania, 1988.

[6] E. J. McShane, "Jensen's inequality," Bulletin of the American Mathematical Society, vol. 43, no. 8, pp. 521-527, 1937.

[7] P. S. Bullen, D. S. Mitrinović, and P. M. Vasić, Means and Their Inequalities, Reidel, Dordrecht, The Netherlands, 1988.

[8] J. Mićić, Z. Pavić, and J. Pečarić, "The inequalities for quasiarithmetic means," Abstract and Applied Analysis, vol. 2012, Article ID 203145, 25 pages, 2012.

[9] Z. Pavić and S. Wu, "Inequalities for convex functions on simplexes and their cones," Abstract and Applied Analysis. In press.

[10] C. P. Niculescu and L.-E. Persson, "Old and new on the HermiteHadamard inequality," Real Analysis Exchange, vol. 29, no. 2, pp. 663-685, 2003.

[11] S. S. Dragomir and C. E. M. Pearce, Selected Topics on HermiteHadamard Inequalities and Applications, RGMIA Monographs, Victoria University, Melbourne, Australia, 2000. 


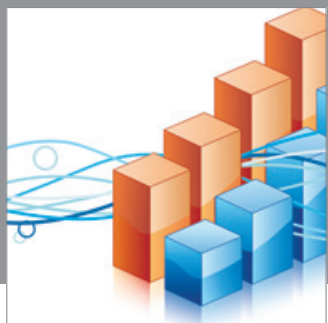

Advances in

Operations Research

mansans

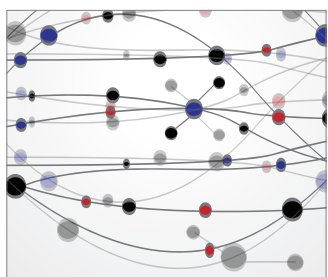

The Scientific World Journal
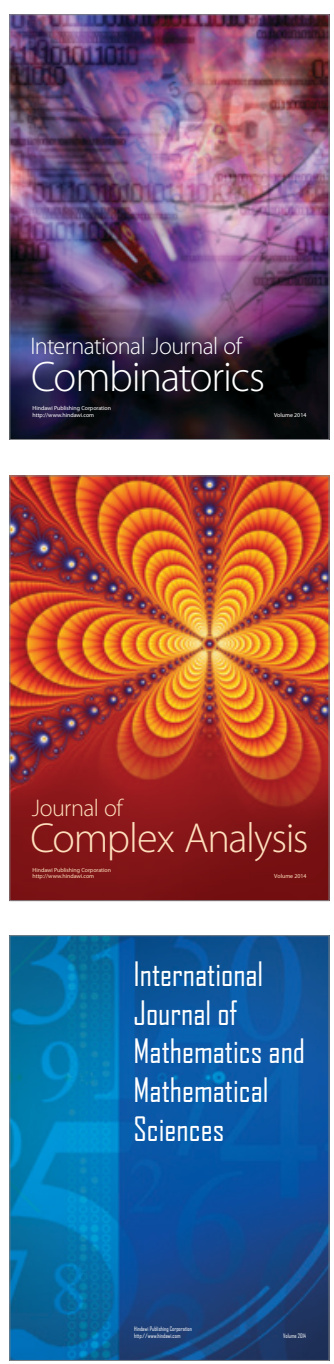
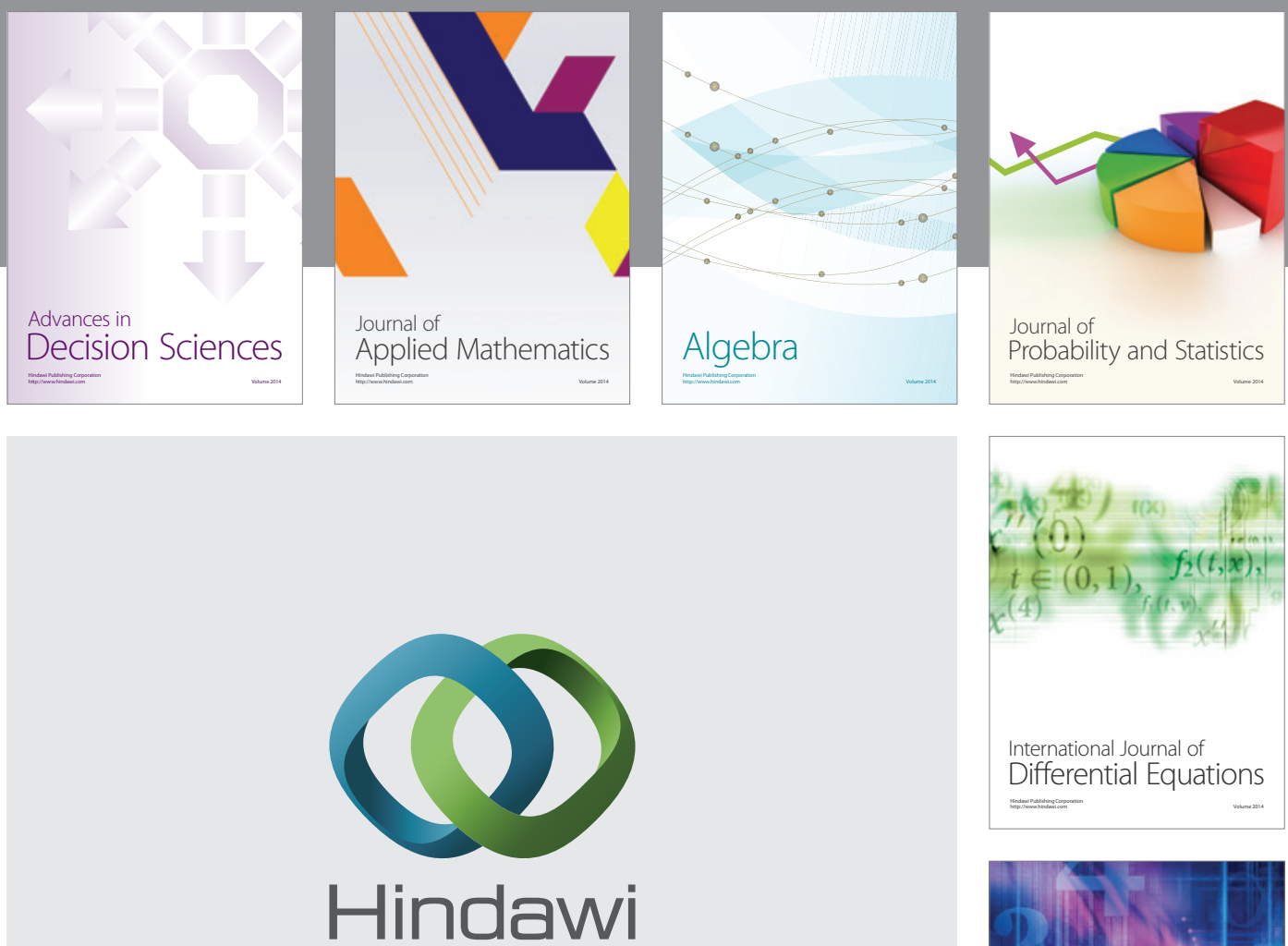

Submit your manuscripts at http://www.hindawi.com
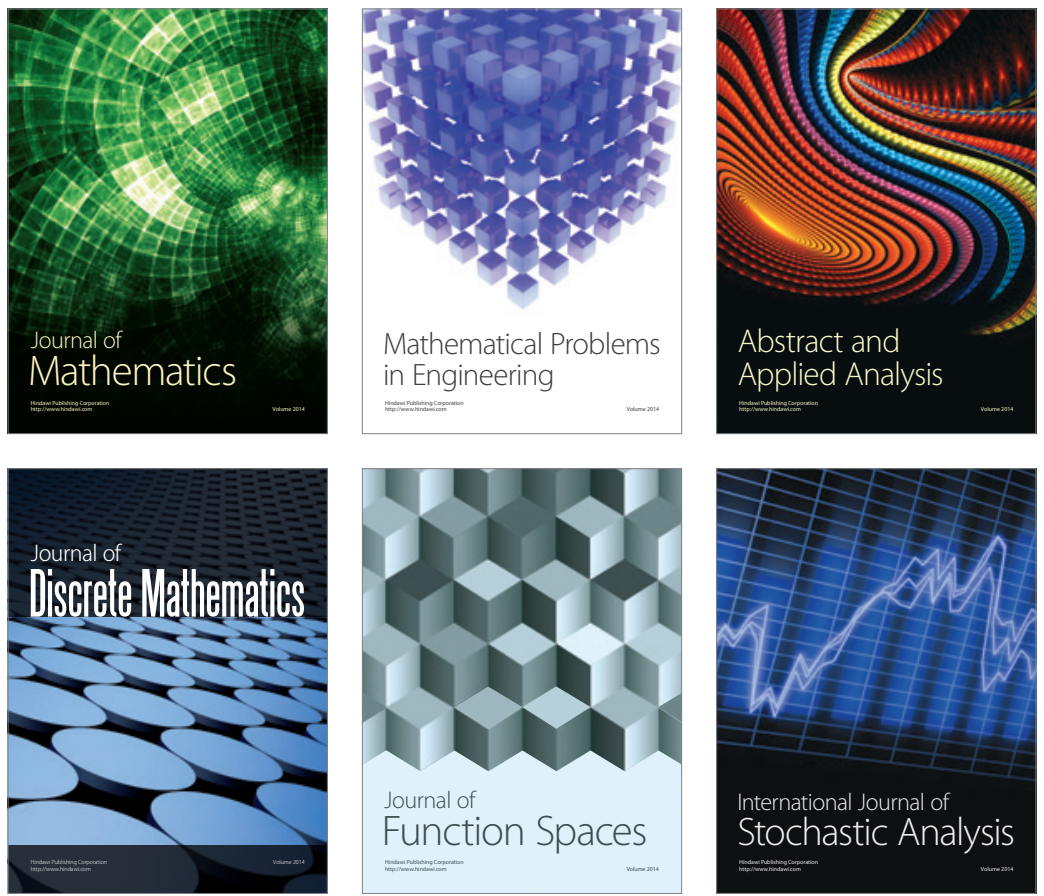

Journal of

Function Spaces

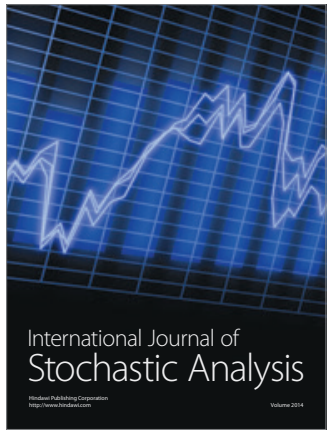

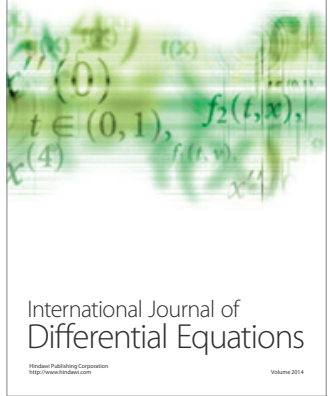
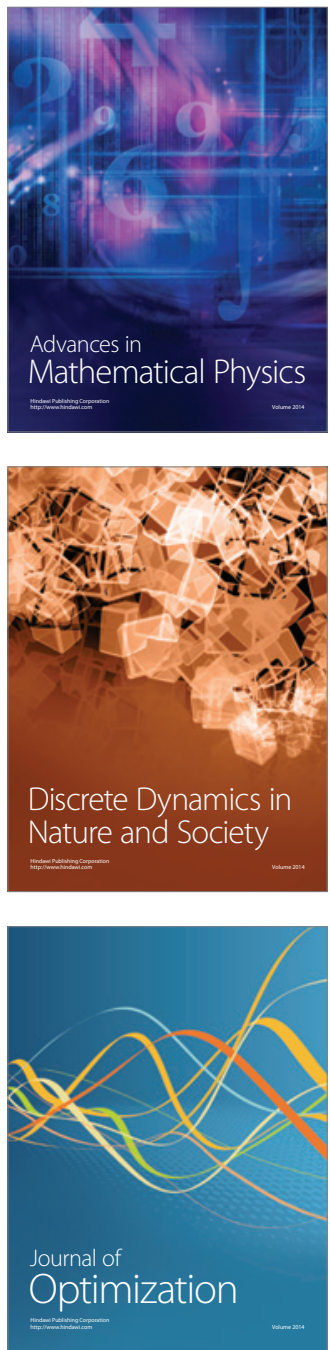